\title{
长脉冲激光等离子体中的密度分布特性
}

\author{
朱莳通 \\ 沈文达 \\ (中国科学院上海光学䢂密机棫研究所) \\ (上海科学技术大学物理系)
}

\section{一、引 女}

最近在激光与等离子体相互作用的研究中，有质动力导致的密度分布修正引起了广大的 实验和理论工作者的兴趣。表征密度分布的上、下平台密度和局域密度标度长度对入射敨光 场的依赖关系都已分别在文献 [1-3]中导出. 然而, 实验和理论的比较表明, 对于 $\mathrm{CO}_{2}$ 激光 辐照的等离子体, 其上平台高度比理论预言的高得多, 其临界密度对场强的依赖性也显示出较 大的分散性 ${ }^{[3]}$. 在文献 [5]中, 我们把临界密度标度长度在大于某一强度时的反常增加归结为 双频或多频激光作用的结果。在本文中，我们将把这现象与密度分布随时间的演化联系起来. 指出在达到准稳态后，上平台密度高度将随激光强度和激光作用时间而增加。临界密度附近 的局域密度标度长度也可能因测量时间的不同而呈现出分散性。

\section{二、五论}

文献 [1] 给出在有质动力作用下,其自洽的密度分布由下面关系式描述:

$$
\begin{aligned}
& \left|A_{s}\right|^{2}-|A|^{2}=2\left(V^{2}-2 \ln V-1\right), \\
& N V=N_{s}=N_{1} V_{1}=N_{2} V_{2},
\end{aligned}
$$

而

$$
\begin{aligned}
2\left(V_{1}^{2}-2 \ln V_{1}-1\right) & =2\left(V_{2}^{2}-2 \ln V_{2}-1\right)-\left|A_{b}\right|^{2}, \\
N_{2} & =\frac{\left|A_{s}\right|^{2}}{4\left(V_{2}-1\right)^{2}},
\end{aligned}
$$

其中 $A=\frac{e E}{m \omega c}, V=v / c_{s}, N=n / n_{c} . n 、 \nu$ 分别是等离子体密度和流速, $n_{c}$ 为临界密度, $c s$ 为离子声速, $e 、 m$ 分别为电子的电荷和质量. $E$ 和 $\omega$ 为电场强度和人射激光的频率, $c$ 为光速. 上面各量的下标 2 表示上平台的量, 下标 1 表示下平台的量, 下标 $s$ 表示声速点的 量.

声速点的电场 $\left|A_{d}\right|$ 和入射敫光电场 $A_{0}$ 之间的关系可以利用文献 [3] 给出的电场解析 表达式

建立, 即

$$
|A|=\left|2 x^{1 / 2}\left(2 k L_{b}\right)^{1 / 3}\left[\zeta /\left(1-N_{0 b}\right)\right]^{1 / 4} A_{0} A_{j}(-z)\right|
$$

$$
\left|A_{s}\right|^{2}=\frac{3.6 A_{0}^{2}}{\sqrt{1-N_{0 b s}}}
$$

本文 1986 年 9 月 18 日收到. 
其中

$$
\begin{aligned}
& \left\{\begin{array}{c}
\frac{2}{3} \zeta^{3 / 2}=\ln \left(1+\sqrt{1-N_{0 b}}\right)-\ln \sqrt{N_{0 b}}-\sqrt{1-N_{0 b}}, \quad\left(N_{0 b}<1\right) \\
\frac{2}{3}(-\zeta)^{3 / 2}=\sqrt{N_{0 b}-1}-\arccos \left(1 / \sqrt{N_{0 b}}\right), \quad\left(N_{0 b}>1\right) \\
z=\left(2 k L_{b}\right)^{2 / 3} \zeta,
\end{array}\right. \\
& k L_{b}=\frac{2^{3 / 2}}{3\left[\sqrt{N_{2}-1}-\arccos \left(1 / \sqrt{N_{2}}\right)\right]},
\end{aligned}
$$

而 $N_{0 b s}$ 由下式决定:

$$
3 k L_{b}\left[\ln \left(1+\sqrt{1-N_{0 b s}}\right)-\ln \sqrt{N_{o b s}}-\sqrt{1-N_{o b s}}\right]=1 .
$$

利用以上这些关系，我们得到上平台的密度 $N_{2}$ 对入射光强度的依赖关系如图 1 曲线 A 所示. 为了与实验和已有的理论比较, 在图 1 中我们还分别用曲线 $\mathrm{B}$ 和 C 示出了 $\mathrm{CO}_{2}$ 激光实验 ${ }^{[1]}$ 和 Lee 等人给出的结果 ${ }^{[1]}$. 可以看到, 当 $k L_{b}$ 满足 (8) 式时, 我们的结果与 Lee 等人的颇为接 近,但在高强度时,与实验结果有较大的偏离.

进一步的分析表明,由于上平台与稀疏波的连结是非稳态的,且连结点是弱间断点（密度 的一阶导数不连续), 因而文献 [3]给出的密度分布实质上是准稳态的密度分布, 对应于 $L_{b}$ 的 ${ }_{b}^{b}$ 表示的时间就是达到准稳态的时间, 随着时间的推移, 准稳态的密度分布将有所改变. 这不 难从(9)和 (6) 式看出. 当 $t$ 取大于 $t_{b}$ 的值时, $L_{t}$ 将代替 $L_{b}$, 从而由 (9) 得出的 $N_{0 b}$ 较小. 当 $A_{0}^{2}$ 固定时,由 (6) 式给出的 $\left|A_{s}\right|^{2}$ 较大,由 (4) 式可知 $N_{2}$ 也较大. 图 1 的曲线 $\mathrm{D}$ 表示了 $k L_{b}=k L_{t}=23$ 时, $N_{2}$ 对入射激光强度的依赖关系, 可以看到它与实验曲线 B 比较接近. 由于 $\mathrm{CO}_{2}$ 激光的脉冲延续时间比钕玻璃长，因而其时间效应将比较明显，使其上平台的高度 值大于 Lee 预言的值, 这就对实验与稳态理论的偏离提供了一个新的合理的解释. 同样的分 析可以解释在较高强度时, 标度长度的反常增加和测得的 $L_{c}$ 值的分散性.

文献 [3] 给出临界密度处的标度长度:

$$
L_{c}=\left|\left(1-N_{s}^{2}\right) / 2 \pi A_{0}^{2} A_{i}\left(-z_{c}\right) A_{i}^{\prime}\left(-z_{c}\right)\right|,
$$

其中 $z_{c}=\left(2 k L_{b}\right)^{2 / 3} \zeta_{c}$.

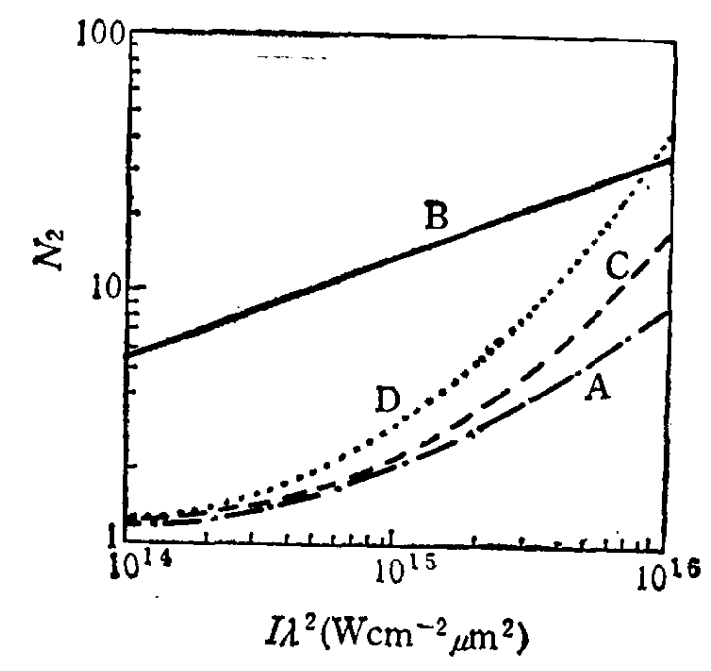

图 $1 N_{2}$ 对 $1 \lambda^{2}$ 的依赖关系 $\left(T_{0}=250 \mathrm{eV}\right)$
A: $k L_{b}=k L_{b}$;
B: 实验曲线[4];
C: Lee 等人预言的曲线; $\mathrm{D}: k L_{b}=k L_{t}=23$

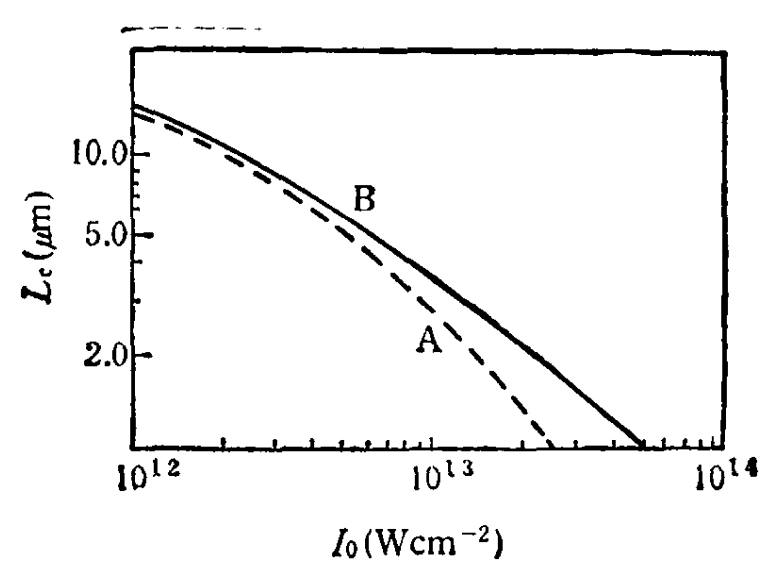

图 $2 L_{c}$ 对 $I_{\mathrm{c}}$ 的依赖关系 A: $k L_{b}=k L_{b} ; \mathrm{B}: k L_{b}=k L_{t}=12.2$ $\left(T_{c}=250 \mathrm{eV}, \lambda=10.6 \mu \mathrm{m}\right)$ 
对于准稳态, 只需用 $L_{t}$ 代替 $L_{b}$. 对于给定的 $A_{0}, L_{c}$ 将随 $L_{t}$ 而变化.

图 2 的曲线 A 表示 $L_{c}$ 在 $k L_{b}-k L_{b}$ 时对 $I_{0}$ 的依赖关系. 曲线 B 则表示 $k L_{b}=$ $\left\{L_{t}=12.2\right.$ 时, $L_{c}$ 对 $I_{0}$ 的依赖关系. 可以看到, 在高强度 $t$ 的变化对 $L_{c}$ 更显著. 如果测 量不是固定在同一时间,则 $L_{c}$ 对 $I_{0}$ 的依赖关系将出现分散性. 如果在较低的人射强度，在 较短的作用时间测量，而在较高强度，在较长的作用时间测量，就会造成 $L_{c}$ 的反常增加. 这 种情况在高强度区域更容易发生. 因而这里对准稳态密度分布时间特性的分析也提供了在实 验中观察到的 $L_{c}$ 异常特性的一个合理解释 ${ }^{[4]}$.

\section{三、结 论}

在激光等离子体相互作用中, 上平台的密度高度可以提供有关影响相互作用机制的重要 信息,而临界密度的标度长度又是决定激光能量的吸收、散射和各种不稳定性的重要参量, 在 理论上给出与实验一致的结果是理论工作的重要任务. 本文考虑到实际的平面电磁波与自由 膨胀等离子体的相互作用过程是一个准稳态的过程, 对自洽的密度分布因受非稳态的自相似 膨胀影响而产生的演化作了修正，它可以合理地解释实验与理论之间的偏离。密度分布的其 他时间特性将在另文中结合其他问题讨论.

[1] Lee, K. et al., Phys. Fluids, 20(1977), 51.

(2] Estabrook, K. et al., Phys. Fluids, 26(1983), 1888.

[ 3] 沈文达、朱莳通, 光学学报, 4(1984), 979 .

[4] Fedosejevs, R. et al., Phys. Fluids, 24(1981), 537.

[ 5 ] 朱莳通、沈文达,物理学报, 35(1986), 882 . 